\title{
Serial Intravascular Ultrasound Assessment of Atherosclerosis Progression and Regression State-of-the-Art and Limitations
}

\author{
Gary S. Mintz, MD; Akiko Maehara, MD
}

\begin{abstract}
Coronary heart disease remains a leading cause of morbidity and mortality. Surrogate imaging endpoints may allow smaller sample sizes and shorter study durations to expedite the process of drug development and testing, and to evaluate potential benefits of novel antiatherosclerotic drugs before clinical endpoint data are available - an approach that may reduce cost and effort. Intravascular ultrasound (IVUS) is particularly suitable because it is readily available and because of its relatively high image resolution, accurate and reproducible measurements, ability to detect mild, angiographically silent atherosclerotic disease that can be a precursor of future coronary events, and suitability for serial (baseline and follow-up) imaging and analysis. However, there are significant limitations to the use of IVUS as an endpoint in progression/regression studies that must be considered when evaluating the results of such studies. (Circ J 2009; 73: 1557-1560)
\end{abstract}

Key Words: Atherosclerosis; Coronary artery disease; Imaging; Intravascular ultrasound; Ischemic heart disease

C oronary heart disease remains a leading cause of morbidity and mortality. Established preventive pharmacological therapies reduce cardiovascular event rates, but only by $30-40 \%$. The assessment of morbidity and mortality as primary endpoints in conventional large-scale clinical trials of established and novel agents is associated with a substantial financial burden. ${ }^{1-3}$ Surrogate endpoints, on the other hand, may allow smaller sample sizes and shorter study durations to expedite the process of drug development and testing, and to evaluate potential benefits of novel anti-atherosclerotic drugs before clinical endpoint data are available - an approach that may reduce cost and effort. ${ }^{1,3-5}$

Intravascular ultrasound (IVUS) is particularly suitable because it is readily available and because of its relatively high image resolution, accurate and reproducible measurements, ability to detect mild, angiographically silent atherosclerotic disease that can be a precursor of future coronary events, and suitability for serial (baseline and follow-up) imaging and analysis. ${ }^{6}$ Conversely, angiographic studies of progression/regression are limited because angiography shows the opacified silhouette of only the lumen; furthermore, the variability of vascular remodeling prevents reliable assessment of the plaque dimensions on the basis of lumen narrowing.

Using planar, not volumetric IVUS analysis, the relation between low-density lipoprotein-cholesterol (LDL-C) levels and the progressive enlargement of coronary plaques was demonstrated in 2003.7 In that study there was a direct linear relationship between changes in left main plaque area plaque area vs LDL-C levels $(\mathrm{r}=0.41, \mathrm{P}<0.0001)$. An LDL-C value of $75 \mathrm{mg} / \mathrm{dl}$ was the threshold below which regression analysis predicted, on average, no increase in atherosclerotic plaque. This observational study also revealed a significant negative correlation between high-density lipoprotein-cholesterol (HDL-C) and atherosclerotic plaque progression; low HDL-C was on average associated with greater plaque progression. During follow-up, adverse nonleft main related cardiovascular events occurred mainly in patients with the greatest rate of left main plaque progression $(\mathrm{P}<0.001){ }^{8}$

\section{State-of-the-Art}

Since then, serial IVUS analysis has become standardized. In contemporary progression/regression studies an untreated $30-40 \mathrm{~mm}$ or longer, moderately diseased untreated coronary artery segment is identified angiographically, a guidewire is positioned into the distal vessel, intracoronary nitroglycerin is administered, the IVUS catheter is inserted over the guidewire into the coronary artery beyond a distal fiduciary point (ie, a well-defined side branch), motorized transducer pullback through a stationary imaging sheath is initiated and continues for at least $30-40 \mathrm{~mm}$ until a welldefined proximal fiduciary point is reached, and then the IVUS catheter is removed. In the core laboratory the lumen and external elastic membrane contours are drawn and areas measured at pre-specified intervals (ie, every $1 \mathrm{~mm}$ ), plaque\&media areas are calculated as external elastic membrane minus lumen, and volumes are calculated using Simpson's rule and then normalized for pullback length. Baseline and follow-up analyses of the entire segment between the proximal and distal fiduciary points are compared as the primary endpoint; comparative analysis of

(Received June 30, 2009; revised manuscript received July 3, 2009; accepted July 5, 2009; released online July 29, 2009)

Cardiovascular Research Foundation, New York City, NY, USA

Mailing address: Gary S. Mintz, MD, Cardiovascular Research Foundation, 111 E 59 th $^{\text {St}}-11^{\text {th }}$ floor, New York, NY 10022, USA. E-mail: gmintz@crf.org

All rights are reserved to the Japanese Circulation Society. For permissions, please e-mail: cj@j-circ.or.jp 
the "worst segment" is reserved for a secondary endpoint because the reproducibility is worse.

Two large-scale pharmacological intervention trials using serial volumetric IVUS analysis studied the effects of statin therapy on coronary plaque progression-regression. ${ }^{9,10}$ The LDL-C threshold that was associated with no disease progression in the earlier, aforementioned observational study was confirmed by the much larger "Reversal of Atherosclerosis with Aggressive Lipid Lowering (REVERSAL) trial", a prospective randomized study that tested the effect of 18 months of intensive vs moderate lipid-lowering therapy on coronary plaque progression. ${ }^{9}$ The "Study to Evaluate the Effect of Rosuvastatin on Intravascular Ultrasound-Derived Coronary Atheroma Burden (ASTEROID)" demonstrated that very high-intensity statin therapy to a mean LDL-C level of $60.8 \mathrm{mg} / \mathrm{dl}$ resulted in overall regression of atherosclerotic plaque; however, there was no control group for comparison. ${ }^{10}$ Third, a small, randomized trial suggested that 5-week infusions of the HDL-mimic, Apo-A1 Milano, induced significant plaque regression in patients with acute coronary syndrome. ${ }^{11}$ Conversely, 2 serial IVUS studies showed no significant difference in plaque progression between patients treated with acyl-coenzyme A cholesterol acyltransferase (ACAT) inhibitors vs placebo; cholesterol esterification by the enzyme ACAT plays an important role in atherosclerotic plaque formation. ${ }^{12,13}$ Thus, negative IVUS studies can prevent needless large-scale clinical trials if negative studies accurately reflect clinical events.

\section{Study Limitations}

Nevertheless, there are significant limitations to those studies that must be considered when evaluating their findings.

Calcium shadows deeper arterial structures. When present, significant calcium precludes identification of the external elastic membrane and, therefore, measurement of atherosclerotic plaque.

Non-uniform rotation distortion, which is an imaging artifact unique to the mechanical, rotating, high-frequency transducers typically used in progression/regression studies, is difficult and sometimes impossible to recognize.

There often are discrepancies between baseline and follow-up pullback lengths that affect volumetric analyses.

Poor imaging techniques necessitating rejection of segments and/or patients can introduce bias.

The most common primary endpoint in IVUS-based progression/regression studies is the absolute change in normalized \% atheroma volume (also called plaque burden) that is calculated as plaque\&media volume divided by external elastic membrane volume over the entire analysis segment. This endpoint has become the "industry standard" because it is associated with the smallest variance. However, changes in \% atheroma volume are small (median of $1.6 \%$ in the pravastatin group and $0.2 \%$ in the atorvastatin group in REVERSAL, median of $-0.8 \%$ in the combined treatment groups of the Apo-A1 Milano study, and median $-0.8 \%$ in ASTEROID). More importantly, population variances are large (standard deviation of $4.9 \%$ in the pravastatin group and $5.1 \%$ in the atorvastatin group in REVERSAL, $3.2 \%$ in the combined treatment groups of the Apo-A1 Milano study, and 3.2\% in ASTEROID); if a study is "positive," slightly more than $50 \%$ regress and slightly less than $50 \%$ progress. This also emphasizes the importance of a control group in all such studies.

Because $\%$ atheroma volume is calculated as plaque\& media volume divided by external elastic membrane volume, it can also be influenced by remodeling; positive remodeling (increase external elastic membrane volume) could reduce $\%$ atheroma volume with no absolute change in plaque mass, and negative remodeling (or an increase in vessel tone) would decrease external elastic membrane volume to increase \% atheroma volume with no absolute change in plaque mass. Several serial IVUS observations have confirmed a broad spectrum of serial remodeling responses (changes in external elastic membrane) in mild-tomoderate atherosclerotic coronary lesions. ${ }^{14,15}$ Schoenhagen et al have observed negative remodeling of the coronary vessel wall during plaque stabilizing therapy that appeared to be related to its antiinflammatory effects. ${ }^{16}$ Schartl et al have showed that positive remodeling was diminished in patients with plaque progression, despite intensive lipidlowering therapy. ${ }^{17}$ Tardif et al concluded that regression of atherosclerotic plaques was accompanied by negative remodeling without an increase in lumen dimensions. ${ }^{18}$ Thus, on the one hand an inward shift of the remodeling pattern may be considered a sign of plaque stabilization, on the other negative remodeling might also affect the primary IVUS endpoint in the absence of actual regression.

IVUS cannot predict which segments will change most (or least) or even which segments will progress or regress, other than the fact that calcified segments change the least.

Arterial segments are selected for analysis based on coronary angiography. These segments may not be representative of segments prone to causing clinical events. Clinical events are most commonly the result of rupture of vulnerable plaques with superimposed thrombus formation. Pathologic studies have shown relatively few "unstable" lesions (ie, vulnerable plaques), even in high-risk patients, ${ }^{19,20}$ making it unlikely that a "blindly" selected $30-40 \mathrm{~mm}$ coronary artery segment based on coronary angiography will, fortuitously, contain even 1 vulnerable plaque. Furthermore, there seems to be a contradiction between the clinical benefit of certain pharmacological interventions, such as statins, and their more limited effect on plaque size. This discrepancy can be explained by stabilization of plaque composition rather than a reduction in plaque mass. However, conventional grayscale IVUS has significant limitations in the assessment of plaque composition and lesion phenotype and their changes over time. Therefore, spectral analysis of IVUS radiofrequency (RF) data was developed to quantify individual coronary plaque components or assess plaque deformability (stability). ${ }^{21-24}$ In a serial RF-IVUS study of patients treated with statins, Kawasaki et al demonstrated stabilization of plaque composition (decrease in lipidic tissue and increase in fibrotic tissue), despite no change in plaque burden. ${ }^{25}$ The Integrated Biomarker And Imaging Study 2 (IBIS 2) trial tested darapladib inhibition of the enzyme lipoprotein-associated phospholipase A2 (Lp-PLA2) on plaque geometry and composition. Necrotic core volume, a key determinant of plaque vulnerability, continued to increase among control patients, but not in patients treated with darapladib; however, the primary endpoint (changes in plaque strain by palpography) was not different between the 2 groups, nor there was a change in plaque burden. ${ }^{26}$ Because its resolution is $100-150 \mu \mathrm{m}$, IVUS with or without $\mathrm{RF}$ analysis is unable to visualize the $<65 \mu \mathrm{m}$ fibrous cap thickness or macrophage infiltration typical of a ruptureprone thin-cap fibroatheroma. Conversely, the superior $10 \mu \mathrm{m}$ resolution of optical coherence tomography (OCT) makes it an ideal technique for identifying these defining 
features of a vulnerable plaque. ${ }^{27-29}$ In a serial OCT imaging study there was a significant increase in fibrous cap thickness during statin therapy. ${ }^{30}$

Finally, a prerequisite for adoption of an imaging technique in progression/regression trials is evidence that this endpoint is a true surrogate for clinical events. Quantitative coronary angiographic studies have shown that progressive obstruction of the coronary lumen is associated with an increased risk of adverse cardiovascular events. ${ }^{31-33}$ Although there are no equivalent IVUS data, an IVUS-based trial reported that intensive antihypertensive therapy with amlodipine reduced coronary plaque progression and adverse cardiovascular events. ${ }^{12}$ The clinical "Pravastatin or Atorvastatin Evaluation and Infection Therapy (PROVE-IT) trial" used the same treatment regimen as REVERSAL and reported a significantly greater reduction in 2-year cardiovascular events in patients with acute coronary syndromes after treatment with intensive vs moderate lipid-lowering therapy.9,34 REVERSAL and PROVE-IT were distinct studies in different patient populations, but when considered together they provide inferential evidence that atherosclerotic progression measured by IVUS would predict an increased risk of cardiovascular events. Conversely, the companion studies Investigation of Lipid Level Management to Understand Its Impact in Atherosclerotic Events (ILLUSTRATE) and Investigation of Lipid Level Management to Understand its Impact in Atherosclerotic Events (ILLUMINATE) showed how complex can be the relationship between IVUS-measured progression/regression and clinical events. These 2 studies compared statins + torcetrapib to statins alone. Although there was no difference in the primary imaging endpoint, the absolute change in \% atheroma volume comparing treatment and control groups in ILLUSTRATE, ${ }^{35}$ ILLUMINATE reported more clinical events including cardiovascular mortality in the treatment group..$^{36}$

\section{Conclusion}

As the global burden of cardiovascular disease increases, there is need for surrogate imaging endpoints to maximize efficiency in the evaluation of new anti-atherosclerotic therapies. Despite its limitations, invasive imaging with IVUS remains the gold standard. RF-based IVUS analysis permits quantitative assessment of atherosclerotic plaque composition to supplement the grayscale IVUS assessment of overall plaque burden, and OCT allows measurement of fibrous cap thickness and detection of macrophages. A single catheter that permits simultaneous imaging with both IVUS (including RF analysis) and OCT during a single pullback is under development. Nevertheless, the definitive link between imaging and clinical endpoints is currently lacking.

\section{Disclosure}

Dr Mintz receives honoraria/consultation fees and grant support from Boston Scientific and Volcano Corporations. Dr Maehara does not report any conflicts.

\section{References}

1. Libby P. The forgotten majority: Unfinished business in cardiovascular risk reduction. J Am Coll Cardiol 2005; 46: 1225-1228.

2. Loscalzo J. Clinical trials in cardiovascular medicine in an era of marginal benefit, bias, and hyperbole. Circulation 2005; 112: 3026 3029 .

3. Böse D, von Birgelen C, Erbel R. Intravascular ultrasound for the evaluation of therapies targeting coronary atherosclerosis. $J$ Am Coll
Cardiol 2007; 49: 925-932.

4. Kastelein JJ, de Groot E. Ultrasound imaging techniques for the evaluation of cardiovascular therapies. Eur Heart J 2008; 29: 849858.

5. Lindsay AC, Choudhury RP. Form to function: Current and future roles for atherosclerosis imaging in drug development. Nat Rev Drug Discov 2008; 7: 517-529.

6. Mintz GS, Nissen SE, Anderson WD, Bailey SR, Erbel R, Fitzgerald $\mathrm{PJ}$, et al. American College of Cardiology clinical expert consensus document on standards for acquisition, measurement and reporting of intravascular ultrasound studies (IVUS). J Am Coll Cardiol 2001; 37: $1478-1492$.

7. von Birgelen C, Hartmann M, Mintz GS, Baumgart D, Schmermund A, Erbel R. Relation between progression and regression of atherosclerotic left main coronary artery disease and serum cholesterol levels as assessed with serial long-term ( $\geq 12$ months) follow-up intravascular ultrasound. Circulation 2003; 108: 2757-2762.

8. von Birgelen C, Hartmann M, Mintz GS, van Houwelingen KG, Deppermann N, Schmermund A, et al. Relationship between cardiovascular risk as predicted by established risk scores versus plaque progression as measured by serial intravascular ultrasound in left main coronary arteries. Circulation 2004; 110: 1579-1585.

9. Nissen SE, Tuzcu EM, Schoenhagen P, Brown BG, Ganz P, Vogel $\mathrm{RA}$, et al; REVERSAL Investigators. Effect of intensive compared with moderate lipid-lowering therapy on progression of coronary atherosclerosis: A randomized controlled trial. JAMA 2004; 291: 1071-1080.

10. Nissen SE, Nicholls SJ, Sipahi I, Libby P, Raichlen JS, Ballantyne $\mathrm{CM}$, et al. Effect of very high-intensity statin therapy on regression of coronary atherosclerosis: The ASTEROID trial. JAMA 2006; 295: $1556-1565$.

11. Nissen SE, Tsunoda T, Tuzcu EM, Schoenhagen P, Cooper CJ, Yasin $\mathrm{M}$, et al. Effect of recombinant ApoA-I Milano on coronary atherosclerosis in patients with acute coronary syndromes: A randomized controlled trial. JAMA 2003; 290: 2292-2300.

12. Nissen SE, Tuzcu EM, Libby P, Thompson PD, Ghali M, Garza D, et al; CAMELOT Investigators, Effect of antihypertensive agents on cardiovascular events in patients with coronary disease and normal blood pressure: The CAMELOT study: A randomized controlled trial. JAMA 2004; 292: 2217-2225.

13. Nissen SE, Tuzcu EM, Brewer HB, Sipahi I, Nicholls SJ, Ganz P, et al. Effect of ACAT inhibition on the progression of coronary atherosclerosis. $N$ Engl J Med 2006; 354: 1253-1263.

14. Von Birgelen C, Hartmann M, Mintz GS, Böse D, Eggebrecht H, Gössl M, et al. Spectrum of remodeling behavior observed with serial long-term ( $\geq 12$ months) follow-up intravascular ultrasound studies in left main coronary arteries. Am J Cardiol 2004; 93: $1107-1113$

15. Sipahi I, Tuzcu EM, Schoenhagen P, Nicholls SJ, Ozduran V, Kapadia $\mathrm{S}$, et al. Compensatory enlargement of human coronary arteries during progression of atherosclerosis is unrelated to atheroma burden: Serial intravascular ultrasound observations from the REVERSAL trial. Eur Heart J 2006; 27: 1664-1670.

16. Schoenhagen P, Tuzcu EM, Apperson-Hansen C, Wang C, Wolski K, Lin $S$, et al. Determinants of arterial wall remodeling during lipidlowering therapy: Serial intravascular ultrasound observations from the Reversal of Atherosclerosis with Aggressive Lipid Lowering Therapy (REVERSAL) trial. Circulation 2006; 113: 2826-2834.

17. Schartl M, Bocksch W, Fateh-Moghadam S. Effects of lipid-lowering therapy on coronary artery remodeling. Coron Artery Dis 2004; 15: $45-51$.

18. Tardif JC, Grégoire J, L'Allier PL, Ibrahim R, Lavoie MA, LeMay $\mathrm{M}$, et al. Effect of atherosclerotic regression on total luminal size of coronary arteries as determined by intravascular ultrasound. Am J Cardiol 2006; 98: 23-27.

19. Burke AP, Virmani R, Galis Z, Haudenschild CC, Muller JE. 34th Bethesda conference: Task force \#2 What is the pathologic basis for new atherosclerosis imaging techniques? J Am Coll Cardiol 2003; 41: $1874-1886$.

20. Cheruvu PK, Finn AV, Gardner C, Caplan J, Goldstein J, Stone GW, et al. Frequency and distribution of thin-cap fibroatheroma and ruptured plaques in human coronary arteries: A pathologic study. J Am Coll Cardiol 2007; 50: 940-949.

21. Kawasaki M, Takatsu H, Noda T, Sano K, Ito Y, Hayakawa K, et al. In vivo quantitative tissue characterization of human coronary arterial plaques by use of integrated backscatter intravascular ultrasound and comparison with angioscopic findings. Circulation 2002; 105: $2487-2492$.

22. Nair A, Kuban BD, Tuzcu EM, Schoenhagen P, Nissen SE, Vince DG. Coronary plaque classification with intravascular ultrasound radio- 
frequency data analysis. Circulation 2002; 106: 2200-2206.

23. Schaar JA, Regar E, Mastik F, McFadden EP, Saia F, Disco C, et al. Incidence of high-strain patterns in human coronary arteries: Assessment with three-dimensional intravascular palpography and correlation with clinical presentation. Circulation 2004; 109: 2716-2719.

24. Okubo M, Kawasaki M, Ishihara Y, Takeyama U, Yasuda S, Kubota $\mathrm{T}$, et al. Tissue characterization of coronary plaques: Comparison of integrated backscatter intravascular ultrasound with virtual histology intravascular ultrasound. Circ J 2008; 72: 1631-1639.

25. Kawasaki M, Sano K, Okubo M, Yokoyama H, Ito Y, Murata I, et al. Volumetric quantitative analysis of tissue characteristics of coronary plaques after statin therapy using three-dimensional integrated backscatter intravascular ultrasound. J Am Coll Cardiol 2005; 45: 1946 1953.

26. Serruys PW, García-García HM, Buszman P, Erne P, Verheye S, Aschermann M, et al. Effects of the direct lipoprotein-associated phospholipase $\mathrm{A}(2)$ inhibitor darapladib on human coronary atherosclerotic plaque. Circulation 2008; 118: 1172-1182.

27. Prati F, Cera M, Ramazzotti V, Imola F, Giudice R, Giudice M, et al. From bench to bedside: A novel technique of acquiring OCT images. Circ J 2008; 72: 839-843.

28. Kataiwa H, Tanaka A, Kitabata H, Imanishi T, Akasaka T. Safety and usefulness of non-occlusion image acquisition technique for optical coherence tomography. Circ J 2008; 72: 1536-1537.

29. Tearney GJ, Yabushita H, Houser SL, Aretz HT, Jang IK, Schlendorf $\mathrm{KH}$, et al. Quantification of macrophage content in atherosclerotic plaques by optical coherence tomography. Circulation 2003; 107: $113-119$.
30. Takarada S, Imanishi T, Kubo T, Tanimoto T, Kitabata H, Nakamura $\mathrm{N}$, et al. Effect of statin therapy on coronary fibrous-cap thickness in patients with acute coronary syndrome: Assessment by optical coherence tomography study. Atherosclerosis 2009; 202: 491-497.

31. Azen SP, Mack WJ, Cashin-Hemphill L, LaBree L, Shircore AM, Selzer RH, et al. Progression of coronary artery disease predicts clinical coronary events: Long-term follow-up from the Cholesterol Lowering Atherosclerosis Study. Circulation 1996; 93: 34-41.

32. Waters D, Craven TE, Lesperance J. Prognostic significance of progression of coronary atherosclerosis. Circulation 1993; 87: 13991401.

33. Ambrose JA, Tannenbaum MA, Alexopoulos D, Hjemdahl-Monsen CE, Leavy J, Weiss M, et al. Angiographic progression of coronary artery disease and the development of myocardial infarction. $J \mathrm{Am}$ Coll Cardiol 1988; 12: 56-62.

34. Cannon CP, Braunwald E, McCabe CH, Rader DJ, Rouleau JL, Belder R, et al. Intensive versus moderate lipid lowering with statins after acute coronary syndromes. $N$ Engl J Med 2004; 350: $1495-$ 1504.

35. Nissen SE, Tardif JC, Nicholls SJ, Revkin JH, Shear CL, Duggan WT, et al; ILLUSTRATE Investigators. Effect of torcetrapib on the progression of coronary atherosclerosis. N Engl J Med 2007; 356: $1304-1316$

36. Barter PJ, Caulfield M, Eriksson M, Grundy SM, Kastelein JJ, Komadja M, et al; ILLUMINATE Investigators. Effects of torcetrapib in patients at high risk for coronary events. N Engl J Med 2007; 357: $2109-2122$. 